\title{
A SHARP ESTIMATE FOR MULTILINEAR MARCINKIEWICZ INTEGRAL OPERATOR*
}

\author{
LIU LANZHE ${ }^{\dagger}$
}

\begin{abstract}
A sharp estimate for multilinear Marcinkiewicz integral operator is obtained. By using this estimate, we obtain the weighted norm inequalities and $L \log L$ type estimate for the multilinear operator.
\end{abstract}

Key words. Multilinear operator; Marcinkiewicz integral operator; Sharp estimate; BMO

AMS subject classifications. 42B20, 42B25

1. Introduction. Let $T$ be a singular integral operator. In[1][2][3], Cohen and Gosselin studied the $L^{p}(p>1)$ boundedness of the multilinear singular integral operator $T^{A}$ defined by

$$
T^{A}(f)(x)=\int_{R^{n}} \frac{R_{m+1}(A ; x, y)}{|x-y|^{m}} K(x, y) f(y) d y .
$$

$\operatorname{In}[8], \mathrm{Hu}$ and Yang obtain a variant sharp estimate for the multilinear singular integral operator. The main purpose of this paper is to prove a sharp estimate for some multilinear operator related to Marcinkiewicz integral operator. As the applications, we obtain the weighted norm inequalities and $L \log L$ type estimate for the multilinear operator.

2. Notations and results. Suppose that $S^{n-1}$ is the unit sphere of $R^{n}(n \geq 2)$ equipped with normalized Lebesgue measure $d \sigma=d \sigma\left(x^{\prime}\right)$. Let $\Omega$ be homogeneous of degree zero and satisfy the following two conditions:

(i) $\Omega(x)$ is continuous on $S^{n-1}$ and satisfies the $\operatorname{Li} p_{\gamma}$ condition on $S^{n-1}(0<$ $\gamma \leq 1)$, i.e.

$$
\left|\Omega\left(x^{\prime}\right)-\Omega\left(y^{\prime}\right)\right| \leq M\left|x^{\prime}-y^{\prime}\right|^{\gamma}, \quad x^{\prime}, y^{\prime} \in S^{n-1} ;
$$

(ii) $\int_{S^{n-1}} \Omega\left(x^{\prime}\right) d x^{\prime}=0$.

Let $m$ be a positive integer and $A$ be a function on $R^{n}$. The multilinear Marcinkiewicz integral operator is defined by

$$
\mu_{\Omega}^{A}(f)(x)=\left[\int_{0}^{\infty}\left|F_{t}^{A}(f)(x)\right|^{2} \frac{d t}{t^{3}}\right]^{1 / 2},
$$

where

$$
F_{t}^{A}(f)(x)=\int_{|x-y| \leq t} \frac{\Omega(x-y)}{|x-y|^{n-1}} \frac{R_{m+1}(A ; x, y)}{|x-y|^{m}} f(y) d y
$$

and

$$
R_{m+1}(A ; x, y)=A(x)-\sum_{|\alpha| \leq m} \frac{1}{\alpha !} D^{\alpha} A(y)(x-y)^{\alpha}
$$

* Received January 30, 2003; accepted for publication August 26, 2004.

${ }^{\dagger}$ College of Mathematics, Changsha University of Science and Technology, Changsha 410077, P.R. of China (lanzheliu@263.net). 
Set

$$
F_{t}(f)(x)=\int_{|x-y| \leq t} \frac{\Omega(x-y)}{|x-y|^{n-1}} f(y) d y
$$

We also define that

$$
\mu_{\Omega}(f)(x)=\left(\int_{0}^{\infty}\left|F_{t}(f)(x)\right|^{2} \frac{d t}{t^{3}}\right)^{1 / 2},
$$

which is the Marcinkiewicz integral operator (see [12]).

Let $H$ be the Hilbert space $H=\left\{h:\|h\|=\left(\int_{0}^{\infty}|h(t)|^{2} \frac{d t}{t^{3}}\right)^{1 / 2}<\infty\right\}$, then for each fixed $x \in R^{n}, F_{t}^{A}(f)(x)$ and $F_{t}(f)(x)$ may be viewed as a mapping from $(0,+\infty)$ to $H$, and it is clear that

$$
\mu_{\Omega}^{A}(f)(x)=\left\|F_{t}^{A}(f)(x)\right\|, \quad \mu_{\Omega}(f)(x)=\left\|F_{t}(f)(x)\right\| .
$$

Note that when $m=0, \mu_{\Omega}^{A}$ is just the commutator generated by Macinkiewicz integral and a function $A$ (see [10][16]). while when $m>0$, it is non-trivial generalizations of the commutator. It has been known that multilinear operators are of great interest in harmonic analysis and have been widely studied by many authors (see [1-5]).

First, let us introduce some notation(see[7][11][13]).

For any locally integrable function $f$, the sharp function of $f$ is defined by

$$
f^{\#}(x)=\sup _{x \in Q} \frac{1}{|Q|} \int_{Q}\left|f(y)-f_{Q}\right| d y,
$$

where, and in what follows, $Q$ will denote a cube with sides parallel to the axes, and $f_{Q}=|Q|^{-1} \int_{Q} f(x) d x$. It is well-known that

$$
f^{\#}(x)=\sup _{x \in Q} \inf _{c \in C} \frac{1}{|Q|} \int_{Q}|f(y)-c| d y .
$$

We say that $f$ belongs to $B M O\left(R^{n}\right)$ if $f^{\#}$ belongs to $L^{\infty}\left(R^{n}\right)$. For $0<r<\infty$, we denote $f_{r}^{\#}$ by

$$
f_{r}^{\#}(x)=\left[\left(|f|^{r}\right)^{\#}(x)\right]^{1 / r} .
$$

Let $M$ be the Hardy-Littlewood maximal operator, that is

$$
M(f)(x)=\sup _{x \in Q} \frac{1}{|Q|} \int_{Q}|f(y)| d y,
$$

we write that $M_{p}(f)=\left(M\left(f^{p}\right)\right)^{1 / p}$, for $k \in N$, we denote by $M^{k}$ the operator $M$ iterated $k$ times, i.e., $M^{1}(f)(x)=M(f)(x)$ and

$$
M^{k}(f)(x)=M\left(M^{k-1}(f)\right)(x) \text { when } k \geq 2 .
$$

Let $B$ be a Young function and $\tilde{B}$ be the complementary associated to $B$, we denote that, for a function $f$,

$$
\|f\|_{B, Q}=\inf \left\{\lambda>0: \frac{1}{|Q|} \int_{Q} B\left(\frac{|f(y)|}{\lambda}\right) d y \leq 1\right\}
$$


and the maximal function by

$$
M_{B}(f)(x)=\sup _{x \in Q}\|f\|_{B, Q}
$$

The main Young function to be using in this paper is $B(t)=t\left(1+\log ^{+} t\right)$ and its complementary $\tilde{B}(t)=\exp t$, the corresponding maximal denoted by $M_{L \log L}$ and $M_{\exp L}$. We have the generalized Hölder's inequality:

$$
\frac{1}{|Q|} \int_{Q}|f(y) g(y)| d y \leq\|f\|_{B, Q}\|g\|_{\tilde{B}, Q}
$$

and the following inequality (in fact they are equivalent), for any $x \in R^{n}$,

$$
M_{L l o g L}(f)(x) \leq C M^{2}(f)(x)
$$

and the following inequalities, for all cube $Q$ and any $b \in B M O\left(R^{n}\right)$,

$$
\left\|b-b_{Q}\right\|_{\exp L, Q} \leq C\|b\|_{B M O}
$$

and

$$
\left|b_{2^{k+1} Q}-b_{2 Q}\right| \leq 2 k|| b \|_{B M O} .
$$

We denote the Muckenhoupt weights by $A_{p}$ for $1 \leq p<\infty$ (see[7]).

Now we are in position to state our results.

Theorem 1. Let $D^{\alpha} A \in B M O\left(R^{n}\right)$ for all $\alpha$ with $|\alpha|=m$. Then for any $0<r<p<1$, there exists a constant $C>0$ such that for any $f \in C_{0}^{\infty}\left(R^{n}\right)$ and any $x \in R^{n}$,

$$
\left(\mu_{\Omega}^{A}(f)\right)_{r}^{\#}(x) \leq C \sum_{|\alpha|=m}\left\|D^{\alpha} A\right\|_{B M O}\left(M_{p}\left(\mu_{\Omega}(f)\right)(x)+M^{2}(f)(x)\right) .
$$

Theorem 2. Let $1<p<\infty, w \in A_{p}$ and $D^{\alpha} A \in B M O\left(R^{n}\right)$ for all $\alpha$ with $|\alpha|=m$. Then $\mu_{\Omega}^{A}$ is bounded on $L^{p}(w)$, that is

$$
\left\|\mu_{\Omega}^{A}(f)\right\|_{L^{p}(w)} \leq C \sum_{|\alpha|=m}\left\|D^{\alpha} A\right\|_{B M O}\|f\|_{L^{p}(w)} .
$$

Theorem 3. Let $w \in A_{1}$ and $D^{\alpha} A \in B M O\left(R^{n}\right)$ for all $\alpha$ with $|\alpha|=m$. Then there exists a constant $C>0$ such that for each $\lambda>0$,

$$
\begin{aligned}
& w\left(\left\{x \in R^{n}: \mu_{\Omega}^{A}(f)(x)>\lambda\right\}\right) \\
& \leq C \sum_{|\alpha|=m}\left\|D^{\alpha} A\right\|_{B M O} \int_{R^{n}} \frac{|f(x)|}{\lambda}\left(1+\log ^{+}\left(\frac{|f(x)|}{\lambda}\right)\right) w(x) d x .
\end{aligned}
$$

REMARK. In Theorem 1, the sharp estimate for $\mu_{\Omega}^{A}$ is given. As in [8][10], Theorem 2 and 3 follow from Theorem 1. So we only need to prove Theorem 1. 
3. Some lemmas. We begin with some preliminary lemmas.

LEmma 1. (Kolmogorov, [7, p.485]) Let $0<p<q<\infty$ and for any function $f \geq 0$. We define that

$$
\begin{aligned}
& \|f\|_{W L^{q}}=\sup _{\lambda>0} \lambda\left|\left\{x \in R^{n}: f(x)>\lambda\right\}\right|^{1 / q}, \\
& N_{p, q}(f)=\sup _{E}\left\|f \chi_{E}\right\|_{L^{p}} p /\left\|\chi_{E}\right\|_{L^{r}},(1 / r=1 / p-1 / q),
\end{aligned}
$$

where the sup is taken for all measurable sets $E$ with $0<|E|<\infty$. Then

$$
\|f\|_{W L^{q}} \leq N_{p, q}(f) \leq(q /(q-p))^{1 / p}\|f\|_{W L^{q}}
$$

Lemma 2. ([11, p.165]) Let $w \in A_{1}$. Then there exists a constant $C>0$ such that for any function $f$ and for all $\lambda>0$,

$$
w\left(\left\{y \in R^{n}: M^{2} f(y)>\lambda\right\}\right) \leq C \lambda^{-1} \int_{R^{n}}|f(y)|\left(1+\log ^{+}\left(\lambda^{-1}|f(y)|\right)\right) w(y) d y .
$$

Lemma 3. ([3, p.448]) Let $A$ be a function on $R^{n}$ and $D^{\alpha} A \in L^{q}\left(R^{n}\right)$ for all $\alpha$ with $|\alpha|=m$ and some $q>n$. Then

$$
\left|R_{m}(A ; x, y)\right| \leq C|x-y|^{m} \sum_{|\alpha|=m}\left(\frac{1}{|\tilde{Q}(x, y)|} \int_{\tilde{Q}(x, y)}\left|D^{\alpha} A(z)\right|^{q} d z\right)^{1 / q}
$$

where $\tilde{Q}$ is the cube centered at $x$ and having side length $5 \sqrt{n}|x-y|$.

LEMma 4. Let $1<p<\infty$ and $D^{\alpha} A \in B M O\left(R^{n}\right)$ for all $\alpha$ with $|\alpha|=m$, $1<r \leq \infty, 1 / q=1 / p+1 / r$. Then $\mu_{\Omega}^{A}$ is bounded from $L^{p}\left(R^{n}\right)$ to $L^{q}\left(R^{n}\right)$, that is

$$
\left\|\mu_{\Omega}^{A}(f)\right\|_{L^{q}} \leq C \sum_{|\alpha|=m}\left\|D^{\alpha} A\right\|_{B M O}\|f\|_{L^{p}}
$$

Proof. By the Minkowski inequality and the condition on $\Omega$, we have

$$
\begin{aligned}
\mu_{\Omega}^{A}(f)(x) & \leq \int_{R^{n}} \frac{|\Omega(x-y)|\left|R_{m+1}(A ; x, y)\right|}{|x-y|^{m+n-1}}|f(y)|\left(\int_{|x-y|}^{\infty} \frac{d t}{t^{3}}\right)^{1 / 2} d y \\
& \leq C \int_{R^{n}} \frac{\left|R_{m+1}(A ; x, y)\right|}{|x-y|^{m+n}}|f(y)| d y .
\end{aligned}
$$

Thus, the lemma follows from [4][5].

4. Proof of Theorems. First, we prove Theorem 1.

Proof of Theorem 1. Fix $\tilde{x} \in R^{n}$. Let $Q=Q\left(x_{0}, l\right)$ be a cube centered at $x_{0}$ and having side length $l$ such that $\tilde{x} \in Q$. It is suffice to prove for $f \in C_{0}^{\infty}\left(R^{n}\right)$ and some constant $C_{0}$, the following inequality holds:

$$
\left(\frac{1}{|Q|} \int_{Q}\left|\mu_{\Omega}^{A}(f)(x)-C_{0}\right|^{r} d x\right)^{1 / r} \leq C\left(M_{p}\left(\mu_{\Omega}(f)\right)(\tilde{x})+M^{2}(f)(\tilde{x})\right) .
$$


Set $\tilde{Q}=10 \sqrt{n} Q$, then $R_{m}(A ; x, y)=R_{m}(\tilde{A} ; x, y)$ and $D^{\alpha} \tilde{A}=D^{\alpha} A-\left(D^{\alpha} A\right)_{\tilde{Q}}$ for $|\alpha|=m$. Let $f_{1}=f \chi_{\tilde{Q}}, f_{2}=f \chi_{R^{n} \backslash \tilde{Q}}$. We write, for $x \in Q$,

$$
\begin{aligned}
F_{t}^{A}(f)(x)= & \int_{|x-y| \leq t} \frac{\Omega(x-y)}{|x-y|^{n-1}} \frac{R_{m}(A ; x, y)}{|x-y|^{m}} f(y) d y \\
& -\sum_{|\alpha|=m} \frac{1}{\alpha !} \int_{|x-y| \leq t} \frac{\Omega(x-y)}{|x-y|^{n-1}} \frac{(x-y)^{\alpha} D^{\alpha} \tilde{A}(y)}{|x-y|^{m}} f_{1}(y) d y \\
& -\sum_{|\alpha|=m} \frac{1}{\alpha !} \int_{|x-y| \leq t} \frac{\Omega(x-y)}{|x-y|^{n-1}} \frac{(x-y)^{\alpha} D^{\alpha} \tilde{A}(y)}{|x-y|^{m}} f_{2}(y) d y
\end{aligned}
$$

then

$$
\begin{aligned}
& \left|\mu_{\Omega}^{A}(f)(x)-\mu_{\Omega}\left(\sum_{|\alpha|=m} \frac{1}{\alpha !} \frac{\left(x_{0}-\cdot\right)^{\alpha}}{\left|x_{0}-\cdot\right|^{m}} D^{\alpha} \tilde{A} f_{2}\right)\left(x_{0}\right)\right| \\
= & || F_{t}^{A}(f)(x)\|-\| \sum_{|\alpha|=m} \frac{1}{\alpha !} \frac{\left(x_{0}-\cdot\right)^{\alpha}}{\left|x_{0}-\cdot\right|^{m}} D^{\alpha} \tilde{A} f_{2}|| \mid \\
\leq & \left\|F_{t}\left(\frac{R_{m}(\tilde{A} ; x, \cdot)}{|x-\cdot|^{m}} f\right)(x)\right\|+\sum_{|\alpha|=m} \frac{1}{\alpha !}\left\|F_{t}\left(\frac{(x-\cdot)^{\alpha}}{|x-|^{m}} D^{\alpha} \tilde{A} f_{1}\right)(x)\right\| \\
& +\sum_{|\alpha|=m} \frac{1}{\alpha !}\left\|F_{t}\left(\frac{(x-\cdot)^{\alpha} D^{\alpha} \tilde{A}}{|x-\cdot|^{m}} f_{2}\right)(x)-F_{t}\left(\frac{\left(x_{0}-\cdot\right)^{\alpha} D^{\alpha} \tilde{A}}{\left|x_{0}-\cdot\right|^{m}} f_{2}\right)\left(x_{0}\right)\right\| \\
\equiv & I(x)+I I(x)+I I I(x),
\end{aligned}
$$

thus,

$$
\begin{aligned}
& \left(\frac{1}{|Q|} \int_{Q}\left|\mu_{\Omega}^{A}(f)(x)-\mu_{\Omega}\left(\sum_{|\alpha|=m} \frac{1}{\alpha !} \frac{\left(x_{0}-\cdot\right)^{\alpha}}{\left|x_{0}-\cdot\right|^{m}} D^{\alpha} \tilde{A} f_{2}\right)\left(x_{0}\right)\right|^{r} d x\right)^{1 / r} \\
\leq & \left(\frac{C}{|Q|} \int_{Q} I(x)^{r} d x\right)^{1 / r}+\left(\frac{C}{|Q|} \int_{Q} I I(x)^{r} d x\right)^{1 / r}+\left(\frac{C}{|Q|} \int_{Q} I I I(x)^{r} d x\right)^{1 / r} \\
\equiv & I+I I+I I I .
\end{aligned}
$$

Now, let us estimate $I, I I$ and $I I I$, respectively. First, using Lemma 3, we have

$$
\begin{aligned}
I & \leq \sum_{|\alpha|=m}\left\|D^{\alpha} A\right\|_{B M O}\left(\frac{1}{|Q|} \int_{Q}\left(\mu_{\Omega}(f)(x)\right)^{p} d x\right)^{1 / p} \\
& \leq C \sum_{|\alpha|=m}\left\|D^{\alpha} A\right\|_{B M O} M_{p}\left(\mu_{\Omega}(f)\right)(\tilde{x})
\end{aligned}
$$


For $I I$, by Lemma 1 and the weak type $(1,1)$ of $\mu_{\Omega}$ (see[6][14]), we have

$$
\begin{aligned}
I I & \leq C \sum_{|\alpha|=m}|Q|^{-1} \frac{\left\|\mu_{\Omega}\left(D^{\alpha} \tilde{A} f_{1}\right) \chi_{Q}\right\|_{L^{r}}}{\left\|\chi_{Q}\right\|_{L^{r /(1-r)}}} \leq C \sum_{|\alpha|=m}|Q|^{-1}\left\|\mu_{\Omega}\left(D^{\alpha} \tilde{A} f_{1}\right)\right\|_{W L^{1}} \\
& \leq C \sum_{|\alpha|=m}|Q|^{-1} \int_{\tilde{Q}}\left|D^{\alpha} \tilde{A}(y)\left\|f(y) \mid d y \leq C \sum_{|\alpha|=m}\right\| D^{\alpha} A\left\|_{\exp L, \tilde{Q}}\right\| f \|_{L \log L, \tilde{Q}}\right. \\
& \leq C \sum_{|\alpha|=m}\left\|D^{\alpha} A\right\|_{B M O} M_{L \log L} f(\tilde{x}) \leq C \sum_{|\alpha|=m}\left\|D^{\alpha} A\right\|_{B M O} M^{2}(f)(\tilde{x}) ;
\end{aligned}
$$

To estimate $I I I$, we write, for $|\alpha|=m$,

$$
\begin{aligned}
& \left\|F_{t}\left(\frac{(x-\cdot)^{\alpha} D^{\alpha} \tilde{A}}{|x-\cdot|^{m}} f_{2}\right)(x)-F_{t}\left(\frac{\left(x_{0}-\cdot\right)^{\alpha} D^{\alpha} \tilde{A}}{\left|x_{0}-\cdot\right|^{m}} f_{2}\right)\left(x_{0}\right)\right\| \\
= & \left(\int_{0}^{\infty} \mid \int_{|x-y| \leq t} \frac{\Omega(x-y)}{|x-y|^{n-1}} \frac{(x-y)^{\alpha} D^{\alpha} \tilde{A}(y)}{|x-y|^{m}} f_{2}(y) d y\right. \\
& \left.-\left.\int_{\left|x_{0}-y\right| \leq t} \frac{\Omega\left(x_{0}-y\right)}{\left|x_{0}-y\right|^{n-1}} \frac{\left(x_{0}-y\right)^{\alpha} D^{\alpha} \tilde{A}(y)}{\left|x_{0}-y\right|^{m}} f_{2}(y) d y\right|^{2} \frac{d t}{t^{3}}\right)^{1 / 2} \\
\leq & \left(\int _ { 0 } ^ { \infty } \left[\int_{|x-y| \leq t,\left|x_{0}-y\right|>t} \frac{|\Omega(x-y)|}{\left.\left.|x-y|^{n-1}\left|f_{2}(y)\right|\left|D^{\alpha} \tilde{A}(y)\right| d y\right]^{2} \frac{d t}{t^{3}}\right)^{1 / 2}}\right.\right. \\
& +\left(\int _ { 0 } ^ { \infty } \left[\int_{|x-y|>t,\left|x_{0}-y\right| \leq t} \frac{\left|\Omega\left(x_{0}-y\right)\right|}{\left.\left.\left|x_{0}-y\right|^{n-1}\left|f_{2}(y)\right|\left|D^{\alpha} \tilde{A}(y)\right| d y\right]^{2} \frac{d t}{t^{3}}\right)^{1 / 2}}\right.\right. \\
& +\left(\int _ { 0 } ^ { \infty } \left[\int_{|x-y| \leq t,\left|x_{0}-y\right| \leq t} \frac{\mid(x-y)^{\alpha} \Omega(x-y)}{|x-y|^{m+n-1}}\right.\right. \\
= & I I I_{1}+I I I_{2}+I I I_{3} .
\end{aligned}
$$

Note that $|x-y| \sim\left|x_{0}-y\right|$ for $x \in \tilde{Q}$ and $y \in R^{n} \backslash \tilde{Q}$. By the condition on $\Omega$, and 
similar to the proof of Lemma 4, we obtain

$$
\begin{aligned}
I I I_{1} & \leq C \int_{R^{n}} \frac{\left|f_{2}(y)\right|\left|D^{\alpha} \tilde{A}(y)\right|}{|x-y|^{n-1}}\left(\int_{|x-y| \leq t<\left|x_{0}-y\right|} \frac{d t}{t^{3}}\right)^{1 / 2} d y \\
& \leq C \int_{R^{n}} \frac{\left|f_{2}(y)\right|\left|D^{\alpha} \tilde{A}(y)\right|}{|x-y|^{n-1}}\left(\frac{1}{|x-y|^{2}}-\frac{1}{\left|x_{0}-y\right|^{2}}\right)^{1 / 2} d y \\
& \leq C \int_{R^{n}} \frac{\left|f_{2}(y)\right|\left|D^{\alpha} \tilde{A}(y)\right|}{|x-y|^{n-1}} \frac{\left|x_{0}-x\right|^{1 / 2}}{|x-y|^{3 / 2}} d y \\
& \leq C \sum_{k=0}^{\infty} \int_{2^{k+1} \tilde{Q} \backslash 2^{k} \tilde{Q}} \frac{|Q|^{1 /(2 n)}}{\left|x_{0}-y\right|^{n+1 / 2}}\left|D^{\alpha} \tilde{A}(y)\right||f(y)| d y \\
& \leq C \sum_{k=0}^{\infty} 2^{-k / 2} \frac{1}{\left|2^{k+1} \tilde{Q}\right|} \int_{2^{k+1} \tilde{Q}}\left(\left|D^{\alpha} \tilde{A}(y)-\left(D^{\alpha} A\right)_{2^{k+1}} \tilde{Q}^{\mid}+\right|\left(D^{\alpha} A\right)_{2^{k+1} \tilde{Q}}-\left(D^{\alpha} A\right)_{\tilde{Q}} \mid\right)|f(y)| d y \\
& \leq C \sum_{k=1}^{\infty} k 2^{-k / 2}\left(|| D^{\alpha} A\left\|_{\exp L, 2^{k} \tilde{Q}}|| f\right\|_{L \log L, 2^{k} \tilde{Q}}+\left\|D^{\alpha} A\right\|_{B M O} M f(\tilde{x})\right) \\
& \leq C \sum_{k=1}^{\infty} k 2^{-k / 2}|| D^{\alpha} A \|_{B M O} M_{L} \log L(f)(\tilde{x}) \\
& \leq C|| D^{\alpha} A \|\left.\right|_{B M O} M^{2}(f)(\tilde{x}) ;
\end{aligned}
$$

Similarly, we have $I I I_{2} \leq C\left\|D^{\alpha} A\right\|_{B M O} M^{2}(f)(\tilde{x})$.

For $\mathrm{III}_{3}$, by the following inequality (see [14]):

$$
\left|\frac{(x-y)^{\alpha} \Omega(x-y)}{|x-y|^{m+n-1}}-\frac{\left(x_{0}-y\right)^{\alpha} \Omega\left(x_{0}-y\right)}{\left|x_{0}-y\right|^{m+n-1}}\right| \leq C\left(\frac{\left|x-x_{0}\right|}{\left|x_{0}-y\right|^{n}}+\frac{\left|x-x_{0}\right|^{\gamma}}{\left|x_{0}-y\right|^{n-1+\gamma}}\right),
$$

we gain

$$
\begin{aligned}
I I I_{3} \leq & C|Q|^{1 / n} \int_{R^{n}} \frac{\left|f_{2}(y)\right|\left|D^{\alpha} \tilde{A}(y)\right|}{\left|x_{0}-y\right|^{n}}\left(\int_{\left|x_{0}-y\right| \leq t,|x-y| \leq t} \frac{d t}{t^{3}}\right)^{1 / 2} d y \\
& +C|Q|^{\gamma / n} \int_{R^{n}} \frac{\left|f_{2}(y)\right|\left|D^{\alpha} \tilde{A}(y)\right|}{\left|x_{0}-y\right|^{n-1+\gamma}}\left(\int_{\left|x_{0}-y\right| \leq t,|x-y| \leq t} \frac{d t}{t^{3}}\right)^{1 / 2} d y \\
\leq & C\left(\int_{R^{n}} \frac{|Q|^{1 / n}\left|D^{\alpha} \tilde{A}(y)\right|}{\left|x_{0}-y\right|^{n+1}}|f(y)| d y+\int_{R^{n}} \frac{|Q|^{\gamma / n}\left|D^{\alpha} \tilde{A}(y)\right|}{\left|x_{0}-y\right|^{n+\gamma}}|f(y)| d y\right) \\
\leq & C \sum_{k=1}^{\infty} k 2^{-k}\left(\left.|| D^{\alpha} A\right|_{\exp L, 2^{k} \tilde{Q}}\|f\|_{L \log L, 2^{k} \tilde{Q}}+\|\left. D^{\alpha} A\right|_{B M O} M(f)(\tilde{x})\right) \\
& +C \sum_{k=1}^{\infty} k 2^{-\gamma k}\left(\left\|\left.D^{\alpha} A\right|_{\exp L, 2^{k} \tilde{Q}}|| f\right\|_{L \log L, 2^{k} \tilde{Q}}+\left\|D^{\alpha} A\right\|_{B M O} M(f)(\tilde{x})\right) \\
\leq & C|| D^{\alpha} A\|\|_{B M O} M^{2}(f)(\tilde{x}) ;
\end{aligned}
$$


Thus,

$$
I I I \leq C \sum_{|\alpha|=m}\left\|D^{\alpha} A\right\|_{B M O} M^{2}(f)(\tilde{x}) .
$$

This completes the proof of Theorem 1.

From Theorem 1 and the weighted boundedness of $\mu_{\Omega}$ and $M$, we may obtain the conclusion of Theorem 2.

From Theorem 1 and Lemma 2, we may obtain the conclusion of Theorem 3.

Acknowledgement. The author would like to express his deep gratitude to the referee for his very valuable comments and suggestions.

\section{REFERENCES}

[1] J. Cohen, A sharp estimate for a multilinear singular integral on $R^{n}$, Indiana Univ. Math. J., 30 (1981), pp. 693-702.

[2] J. Cohen And J. Gosselin, On multilinear singular integral operators on $R^{n}$, Studia Math., 72 (1982), pp. 199-223.

[3] J. Cohen And J. Gosselin, A BMO estimate for multilinear singular integral operators, Illinois J. Math., 30 (1986), pp. 445-465.

[4] Y. DiNG, A note on multilinear fractional integrals with rough kernel, Adv. in Math. (China), 30 (2001), pp. 238-246.

[5] Y. Ding AND S.Z. Lu, Weighted boundedness for a class rough multilinear operators, Acta Math. Sinica, 17 (2001), pp. 517-526.

[6] Y. Ding, S.Z. Lu And Q. Xue, On Marcinkiewicz integral with homogeneous kernels, J. Math. Anal. Appl., 245 (2000), pp. 471-488.

[7] J. Garcia-Cuerva and J.L. Rubio de Francia, Weighted norm inequalities and related topics, North-Holland Math. Stud., 16, pp. Amsterdam, 1985.

[8] G. Hu AND D.C. YANG, A variant sharp estimate for multilinear singular integral operators, Studia Math., 141 (2000), pp. 25-42.

[9] L.Z. LiU, Boundedness for multilinear Marcinkiewicz Operators on certain Hardy Spaces, Inter. J. of Math. and Math. Sci., 2 (2003), pp. 87-96.

[10] L.Z. LiU, The continuity of commutators on Triebel-Lizorkin spaces, Integral Equations and Operator Theory, 49 (2004), pp. 65-75.

[11] C. PeREz, Endpoint estimate for commutators of singular integral operators, J. Func. Anal., 128 (1995), pp. 163-185.

[12] C. Perez, Weighted norm inequalities for singular integral operators, J. London Math. Soc., 49 (1994), pp. 296-308.

[13] C. Perez and G. Pradolini, Sharp weighted endpoint estimates for commutators of singular integral operators, Michigan Math. J., 49 (2001), pp. 23-37.

[14] M. Sakamoto and K. Yabute, Boundedness of Marcinkiewicz functions, Studia Math, 135 (1999), pp. 103-142.

[15] A. Torchinsky, The real variable methods in harmonic analysis, Pure and Applied Math, 123, pp. Academic Press, New York, 1986.

[16] A. Torchinsky And S. Wang, A note on the Marcinkiewicz integral, Colloq. Math., 60/61 (1990), pp. 235-243. 